\title{
Functional respiratory imaging to assess the interaction between systemic roflumilast and inhaled ICS/LABA/LAMA
}

This article was published in the following Dove Press journal:

International Journal of COPD

4 February 2016

Number of times this article has been viewed

\author{
Wim Vos' \\ Bita Hajian² \\ Jan De Backer' \\ Cedric Van Holsbeke' \\ Samir Vinchurkar ${ }^{\prime}$ \\ Rita Claes ${ }^{2}$ \\ Annemie Hufkens ${ }^{2}$ \\ Paul M Parizel ${ }^{3}$ \\ Lieven Bedert ${ }^{4}$ \\ Wilfried De Backer ${ }^{2}$ \\ 'FLUIDDA nv, Groeningenlei, Kontich, \\ ${ }^{2}$ Department of Respiratory Medicine, \\ ${ }^{3}$ Department of Radiology, University \\ Hospital Antwerp, Wilrijkstraat, \\ Edegem, ${ }^{4}$ Department of Respiratory \\ Medicine, ZNA Middelheim Hospital, \\ Antwerp, Belgium
}

Correspondence: Wim Vos FLUIDDA nv, Groeningenlei I32, 2550 Kontich, Belgium

$\mathrm{Tel}+3234508720$

Fax +3234508729

Email wim.vos@fluidda.com
Background: Patients with COPD show a significant reduction of the lobar hyperinflation at the functional residual capacity level in the patients who improved $>120 \mathrm{~mL}$ in forced expiratory volume in 1 second $\left(\mathrm{FEV}_{1}\right)$ after 6 months of treatment with roflumilast in addition to inhaled corticosteroids (ICSs)/long-acting beta-2 agonists (LABAs)/long-acting muscarinic antagonists (LAMAs)

Methods: Functional respiratory imaging was used to quantify lobar hyperinflation, blood vessel density, ventilation, aerosol deposition, and bronchodilation. To investigate the exact mode of action of roflumilast, correlations between lobar and global measures have been tested using a mixed-model approach with nested random factors and Pearson correlation, respectively.

Results: The reduction in lobar hyperinflation appears to be associated with a larger blood vessel density in the respective lobes $(t=-2.154, P=0.040)$; lobes with a higher percentage of blood vessels reduce more in hyperinflation in the responder group. Subsequently, it can be observed that lobes that reduce in hyperinflation after treatment are better ventilated $(t=-5.368, P<0.001)$. Functional respiratory imaging (FRI)-based aerosol deposition showed that enhanced ventilation leads to more peripheral particle deposition of ICS/LABA/LAMA in the better-ventilated areas $(t=2.407$, $P=0.024)$. Finally, the study showed that areas receiving more particles have increased FRI-based bronchodilation ( $t=2.564, P=0.017)$, leading to an increase in $\mathrm{FEV}_{1}(R=0.348, P=0.029)$.

Conclusion: The study demonstrated that orally administered roflumilast supports the reduction of regional hyperinflation in areas previously undertreated by inhalation medication. The local reduction in hyperinflation induces a redistribution of ventilation and aerosol deposition, leading to enhanced efficacy of the concomitant ICS/LABA/LAMA therapy. FRI appears to be a sensitive tool to describe the mode of action of novel compounds in chronic obstructive pulmonary disease. Future studies need to confirm the enhanced sensitivity and the potential of FRI parameters to act as surrogates for clinically relevant, but more difficult to measure, end points such as exacerbations.

Keywords: modeling, physiology, ventilation, aerosol distribution, FRI

\section{Introduction}

Today, the standard of care for patients suffering from chronic obstructive pulmonary disease (COPD) usually consists of long-acting beta-2 agonists (LABAs), long-acting muscarinic antagonists (LAMAs), inhaled corticosteroids (ICSs), or a combination of these. Recently, a phosphodiesterase type 4 (PDE4) inhibitor has been added as a therapeutic option, but some open questions remain. While LABAs and LAMAs relax the smooth muscle, the ICS component and PDE4 inhibitor are targeted at reducing the chronic inflammation associated with COPD. While ICS combined with LABA remains an established standard, several monoclonal antibodies, a new 
class of anti-inflammatory agents, are under development to treat respiratory diseases. ${ }^{1,2}$ Often, these products are administered systemically either orally or intravenously. During the clinical trials investigating the efficacy of these novel compounds, it is of paramount importance to understand the interaction between the systemic and the inhaled drugs as it is expected that the systemic drugs will only gradually replace the inhaled therapy, if at all. This implies that patients in clinical practice will be treated with both inhaled and systemic drugs, which is already often the case. So far, traditional end points, such as the forced expiratory volume in 1 second $\left(\mathrm{FEV}_{1}\right)$, and exacerbations have been typically used to assess the efficacy of novel drugs. However, for both of these end points, significant challenges arise when applied to the study of anti-inflammatory drugs. The FEV alone lacks the sensitivity to describe regional effects of the novel therapy, which for an anti-inflammatory product is a crucial aspect of the mechanism of action. While exacerbations are clinically relevant end points, the lack of a specific definition introduces significant variability, especially in multicenter trials. In addition, to detect a beneficial effect on exacerbations, the investigators first have to establish a baseline exacerbation rate to assess potential improvements by the new drug. Both establishing the baseline and assessing the therapeutic effect require long and large clinical trials. Therefore, the development of novel drugs would benefit from additional end points that yield regional information and that could potentially act as surrogates for exacerbations in early Phase II trials. The latter would increase the probability of success of the, often-expensive, Phase III trials. Functional respiratory imaging (FRI) is a novel tool consisting of a combination of high-resolution computed tomography (HRCT) and computational fluid dynamics (CFD). The Supplementary video gives a short summary of how functional respiratory imaging works. The HRCT provides regional information on the lung, airway, and vascular structures, while the CFD subsequently provides the functional component in terms of airway resistance and aerosol deposition characteristics. The regional ventilation and deposition were previously validated on a lobar basis using single-photon emission computed tomography (SPECT), ${ }^{3}$ gamma scintigraphy, ${ }^{4}$ and hyperpolarized gases. ${ }^{5}$ Subsequently, the method has been used to investigate the mechanism of action of LABAs, ${ }^{6}$ LABAs/ICSs, ${ }^{7}$ short-acting beta-2 agonists/short-acting muscarinic antagonists, ${ }^{8}$ and antioxidant therapy. ${ }^{9}$

In a recent study, ${ }^{10}$ our group assessed the effect of the PDE4 inhibitor roflumilast as add-on therapy to ICSs/ LABAs/LAMAs in Global initiative for chronic Obstructive
Lung Disease (GOLD) stages III and IV COPD patients. Roflumilast is a selective PDE4 inhibitor. ${ }^{11}$ PDE4 regulates cyclic adenosine monophosphate (cAMP) in most of the cell types that are involved in inflammatory processes. Inhibition of PDE4 reduces the breakdown of cAMP, which in turn downregulates the inflammatory process. Earlier studies ${ }^{12,13}$ have confirmed the anti-inflammatory capabilities of the compound in terms of reducing the level of circulating tumor necrosis factor (TNF)- $\alpha$, reducing the level of TNF- $\alpha$ in bronchoalveolar lavage (which acts as a proinflammatory mediator), and increasing the level of interleukin-10, an antiinflammatory mediator. Two large-scale clinical trials ${ }^{14,15}$ have assessed the clinical efficacy of roflumilast in terms of improving or stabilizing $\mathrm{FEV}_{1}$ and reducing exacerbations. The present study used a combination of classical end points and FRI demonstrated a superior effect of roflumilast relative to placebo. At the same time, the trial demonstrated that especially patients suffering from dynamic hyperinflation at baseline (eight out of 23 patients, 35\%), determined using the 6-minute walk test, benefited from roflumilast, with a posttreatment improvement in $\mathrm{FEV}_{1}>120 \mathrm{~mL}$. In the discussion of that manuscript, we speculated that this was caused by the fact that the orally administered roflumilast reached areas in the lung previously undertreated by ICS. This study aims to extend the research by further investigating this hypothesis in the responder group. Specifically, the regional vasculature and the deposition patterns of the inhaled triple therapy are analyzed in more detail using FRI. The hypothesis for the mode of action of roflumilast as outlined in Figure 1 will be assessed. This study therefore focuses on assessing regional hyperinflation, internal airflow distribution, regional aerosol deposition patterns, and image-based bronchodilation.

\section{Orally administered roflumilast reaches areas undertreated with inhaled medication}

\section{Reduction in regional hyperinflation}

Redistribution of internal airflow distribution

Redistribution of inhaled ICS/LABA/LAMA

Additional improvement in $\mathrm{FEV}_{1}$, exercise tolerance,...

Figure I Hypothesis for the mode of action of roflumilast.

Abbreviations: $\mathrm{FEV}_{1}$, forced expiratory volume in I second; ICS, inhaled corticosteroid; LABA, long-acting beta-2 agonist; LAMA, long-acting muscarinic antagonist. 


\section{Materials and methods}

The study design can be found in De Backer et $\mathrm{a}^{10}$ and is summarized in the CONsolidated Standards Of Reporting Trials (CONSORT) diagram in Figure 2. Baseline measurement consisted of spirometry, body plethysmography, 6-minute walk test, patient reported outcome parameters, and FRI. All measurements were repeated after 6 months of treatment. Both baseline and 6-month measurements were performed after bronchodilation. The remainder of this article will mainly discuss the parameter $\mathrm{FEV}_{1}$, which is measured using spirometry and a set of FRI-based parameters, which are extensively described in the following sections. FRI parameters are derived from HRCT scans taken at functional residual capacity (FRC) and total lung capacity (TLC) at baseline and after 6 months of treatment. Patient characteristics and demographics can be found in Table 1. The study was approved by the ethical committee of the University Hospital of Antwerp, and written informed consent was obtained from each participant. ClinicalTrials.gov Identifier: NCT01480661.

\section{Computed tomography image acquisition}

The level of inspiration during computed tomography (CT) was monitored with a commercially available spirometry system (Spirostik; Geratherm Respiratory GmbH, Kissingen,
Germany) that enables real-time monitoring of the breathing cycle. This ensured that the examinations were performed at the correct lung volume. The CT settings were as follows: tube voltage, $100 \mathrm{kV}$; tube current, $10-200 \mathrm{mAs}$; noise factor, 45; collimation, $0.625 \mathrm{~mm}$; rotation time, 0.6 seconds; and pitch factor, 1.375.

\section{FRI assessment of blood vessel density}

Blood vessel density can be determined through segmentation and three-dimensional reconstruction of the blood vessels. The segmentation is based on a Hounsfield unit (HU) threshold of $[-600 ; 600]$ and is performed on the TLC scan. The blood vessel density can be considered a surrogate for perfusion, as outlined in the study by De Backer et al. ${ }^{10}$ Because roflumilast is orally administered, the regional perfusion determines the regional delivery of the PDE4 inhibitor.

\section{FRI assessment of regional hyperinflation}

The FRI process includes segmentation of the lung volumes at FRC and TLC from the HRCT images by using an HU threshold of $[-1,024 ;-400]$. In addition, the fissures that separate the individual lung lobes are identified. By using the fissure lines as cutting planes, the individual lobe volumes can be determined and expressed as percentages of predicted values (Figure 3).

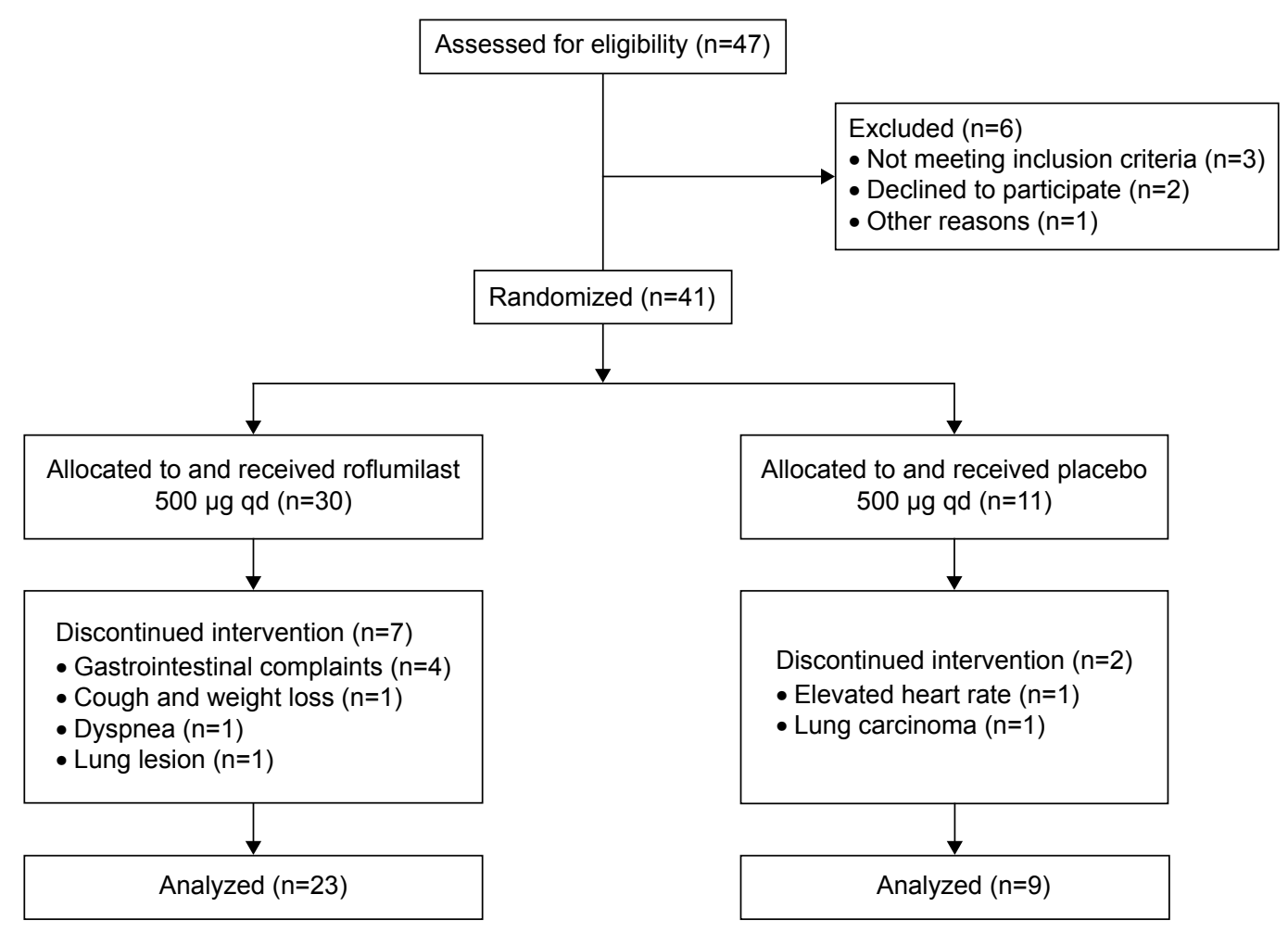

Figure 2 CONSORT diagram of the clinical trial.

Abbreviations: CONSORT, CONsolidated Standards Of Reporting Trials; qd, once daily. 
Table I Patient characteristics and demographics after randomization

\begin{tabular}{|c|c|c|c|c|}
\hline & \multicolumn{3}{|l|}{ Roflumilast } & \multirow[t]{2}{*}{ Placebo } \\
\hline & Total & Responders & Nonresponders & \\
\hline Length $(\mathrm{cm})$ & $166.2 \pm 6.44$ & $166.75 \pm 7.77$ & $165.90 \pm 5.90$ & $170.06 \pm 10.56$ \\
\hline Pack-years smoking (years) & $53.13 \pm 35.57$ & $73.94 \pm 46.99$ & $42.03 \pm 22.48$ & $54.83 \pm 30.24$ \\
\hline Age (years) & $63.61 \pm 7.38$ & $62.12 \pm 6.94$ & $64.40 \pm 7.73$ & $70 \pm 6.76$ \\
\hline Weight (kg) & $84.08 \pm 28.58$ & $87.90 \pm 35.45$ & $82.04 \pm 25.35$ & $92.8 I \pm 26.18$ \\
\hline FVC (\%pred) & $79.76 \pm 21.08$ & $82.24 \pm 23.62$ & $78.44 \pm 20.35$ & $79.97 \pm 13.43$ \\
\hline FEV (\%pred) & $41.3 \pm 12.17$ & $46.91 \pm 11.04$ & $38.31 \pm 12.01$ & $47.28 \pm 11.19$ \\
\hline FEV,/FVC (\%) & $42.5 \pm 12.62$ & $47.64 \pm 12.33$ & $39.77 \pm 12.29$ & $46.6 I \pm 10.4 \mid$ \\
\hline RV (\%pred) & $|7| .32 \pm 35.3 \mid$ & $|68.2| \pm 24.48$ & $172.97 \pm 40.64$ & $146.36 \pm 28.26$ \\
\hline TLC (\%pred) & $114.44 \pm 21.39$ & $112.26 \pm 20.69$ & $\mid 15.61 \pm 22.39$ & $106.22 \pm 11.02$ \\
\hline FRC (\%pred) & $147.22 \pm 33.72$ & $144.88 \pm 26.69$ & $\mid 48.47 \pm 37.76$ & $130.94 \pm 25.07$ \\
\hline Raw (kPas/L) & $0.77 \pm 0.3$ & $0.7 I \pm 0.24$ & $0.80 \pm 0.33$ & $0.67 \pm 0.35$ \\
\hline sRaw (kPas) & $3.97 \pm 1.96$ & $3.43 \pm 0.91$ & $4.24 \pm 2.30$ & $3 \pm 1.26$ \\
\hline $\mathrm{LCl}$ (units) & $8.87 \pm 1.33$ & $8.63 \pm 1.21$ & $9.02 \pm 1.44$ & $9.16 \pm 1.94$ \\
\hline Nitrogen washout time (min) & $5.48 \pm 2.51$ & $5.22 \pm 2.66$ & $5.64 \pm 2.56$ & $3.46 \pm 1.16$ \\
\hline 6MWT (m) & $357.53 \pm 90.02$ & $393.83 \pm 84.48$ & $340.77 \pm 90.66$ & $403.88 \pm 148.84$ \\
\hline
\end{tabular}

Note: Data presented as mean \pm standard deviation.

Abbreviations: 6MWT, 6-minute walk test; \%pred, percentage of predicted; FEV , forced expiratory volume in I second; FRC, functional residual capacity; FVC, forced vital capacity; LCl, lung clearance index; Raw, airways resistance; RV, residual volume; sRaw, specific airways resistance; TLC, total lung capacity.

\section{FRI assessment of internal airflow distribution}

The patient's specific internal airflow distribution can be determined by subtracting the FRC lobe volume from the TLC lobe volume.

\section{FRI assessment of regional aerosol deposition}

Regional aerosol deposition is determined by simulating the flow in the patient-specific geometries using patient-specific boundary conditions by means of CFD. CFD essentially solves the Navier-Stokes equations numerically on a computational grid. While solving the flow equations particles are released simultaneously in the flow, and the force mass balance of the individual particles is determined through additional discrete phase computations. More information on the method can be found in the study by De Backer et al. ${ }^{16}$ The patient-specific HRCT-based airway geometries constitute the basis for the aerosol deposition assessments. The three-dimensionally reconstructed airway geometries were transformed into computational grids using TGrid 14.0 (Ansys Inc., Canonsburg, PA, USA). High-quality, unstructured grids consisting of tetrahedral elements were created. Several grids were tested to assess the mesh sensitivity, and a typical grid of $3 \times 10^{6}$ cells was selected for the analyses to ensure good resolution at all high-gradient regions.

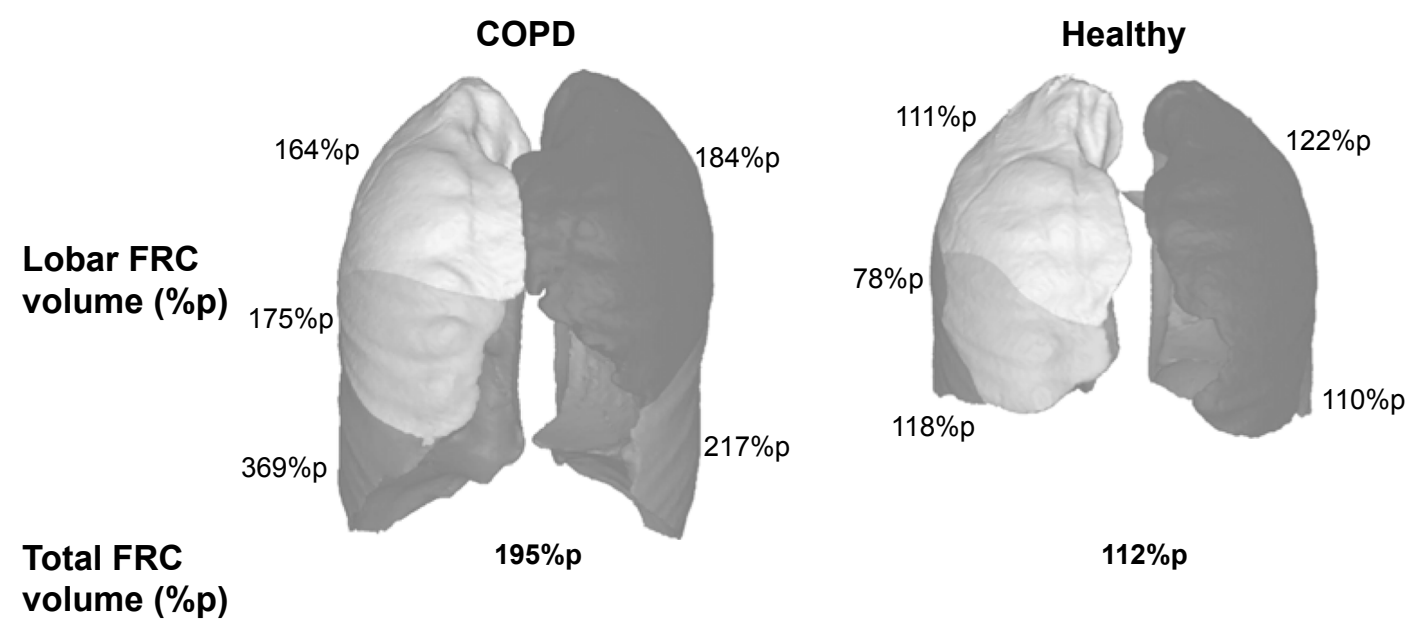

Figure 3 Regional hyperinflation at the FRC level for a COPD patient from the roflumilast trial and a healthy volunteer (both female, both I70 $\mathrm{cm}$ in height). Abbreviations: \%p, percentage of predicted; COPD, chronic obstructive pulmonary disease; FRC, functional residual capacity. 
Flow simulations were performed using Fluent 14.0 (Ansys Inc.). The air in the lower airway was considered to be homogeneous, incompressible, and Newtonian. The flow simulations use so much patient-specific information (eg, airway geometry, internal airflow distribution, inhalation profile) that they provide an accurate idea of the regional resistance and deposition patterns. These profiles were determined in the clinical center by means of a pneumotachometer. The pressure-based solver was used with a node-based GreenGauss gradient treatment. A second-order pressure discretization scheme was selected for the pressure calculation, and a second-order upwind scheme was used for the momentum equations. The pressure-velocity coupling was solved using the Semi-Implicit Method for Pressure-Linked Equations (SIMPLE) algorithm. Turbulence was evaluated through large eddy simulations with a turbulent kinetic subgrid model to assess the possible influence of turbulence on the flow pattern and aerosol deposition. Aerosol transport was modeled by an implicit Runge-Kutta Lagrangian discrete particle model, with a one-way coupling of the forces from the flow to the particle and taking into account the Saffman lift forces. Particles were considered deposited the moment they hit the airway wall.

The aerosol characteristics used for the simulations include the mass mean aerodynamic diameter (MMAD), the geometric standard deviation (GSD), and the fine particle fraction (FPF) of the respective ICS/LABA and LAMA products. Table 2 provides an overview of the aerosol parameters. Inhalation regimens in the trial were as follows:

Table 2 Aerosol characteristics of LAMA and LABA/ICS used in the FRI deposition calculations

\begin{tabular}{|c|c|c|c|}
\hline Inhaler & MMAD & GSD & FPF (\%) \\
\hline Spiriva (LAMA) ${ }^{26}$ & 3.9 & 1.8 & 23.21 \\
\hline Onbrez (LABA) $)^{26}$ & 3.2 & 2 & 39.41 \\
\hline Oxis $(\mathrm{LABA})^{27}$ & 2.13 & 1.72 & 69.06 \\
\hline Pulmicort (ICS) $)^{28}$ & 1.9 & 2.1 & 48 \\
\hline \multicolumn{4}{|c|}{ Seretide Diskus (ICS/LABA) ${ }^{29}$} \\
\hline ICS & 3.54 & 1.49 & 21.6 \\
\hline LABA & 3.57 & 1.46 & 23.34 \\
\hline \multicolumn{4}{|c|}{ Symbicort (ICS/LABA) $)^{29}$} \\
\hline ICS & 3.30 & 1.57 & 38.71 \\
\hline LABA & 3.09 & 1.56 & 43.73 \\
\hline \multicolumn{4}{|c|}{ Inuvair MDI (ICS/LABA) $)^{30}$} \\
\hline ICS & 1.3 & 1.97 & 38.07 \\
\hline LABA & 1.3 & 2.00 & 39.47 \\
\hline \multicolumn{4}{|c|}{ Seretide MDI (ICS/LABA) $)^{31}$} \\
\hline ICS & 3.5 & 1.9 & 42.06 \\
\hline LABA & 3.4 & 1.9 & 42.94 \\
\hline
\end{tabular}

Abbreviations: FPF, fine particle fraction; FRI, functional respiratory imaging; GSD, geometric standard deviation; ICS, inhaled corticosteroid; LABA, long-acting beta-2 agonist; LAMA, long-acting muscarinic antagonist; MDI, metered dose inhaler; MMAD, mass mean aerodynamic diameter.
Spiriva 18 (100\% of the patients), Seretide Diskus 50/500 and 50/250 (54\% and 4\%), Symbicort $9 / 320$ and 4.5/160 (18\% and 7\%), Inuvair $12 / 200$ and $6 / 100$ (11\% and $4 \%$ ), Pulmicort 400 and 200, Oxis 9, Onbrez 150, and Seretide MDI 25/250 (all 4\%).

\section{FRI assessment of bronchodilation}

Segmentation of the airways or image-based airway volume can be performed down to the level of the smaller airways with a diameter of 1-2 mm using an HU threshold of $[-1,024 ;-824]$. By performing airway segmentation at baseline and at 6 months, the patient-specific three-dimensional geometry of the airways can be extracted at both time points. The two geometries can be subsequently placed on top of each other, and regional measurements of changes in airway volume (ie, bronchodilation or bronchoconstriction) can be performed. FRI typically reports changes through color-coded figures.

\section{Statistics}

Statistical analysis was performed using the open-source statistical environment R 3.02. Correlations between parameters that are measured on a lobar basis were assessed using a mixed-model approach, wherein the different lobes were considered to be nested random factors. Correlations between global measurements were determined using Pearson correlation. Significance level was set at $P<0.05$. A responder group was a priori defined based on the measurement error of $\mathrm{FEV}_{1}$, which was recently determined to be $120 \mathrm{~mL} \cdot{ }^{17}$

\section{Results}

De Backer et $\mathrm{a} \mathrm{l}^{10}$ previously reported a significant reduction of the lobar hyperinflation (iLobes_FRC) at the FRC level in the patients who improved $>120 \mathrm{~mL}$ in $\mathrm{FEV}_{1}$, the so-called responder group. The reduction in lobar hyperinflation appears to be associated with a larger blood vessel density in the respective lobes (Figure 4; $t=-2.154, P=0.040$ ): lobes with a higher percentage of blood vessels reduce more during hyperinflation in the responder group. No correlation between blood vessel density and change in hyperinflation is found in the placebo group (Figure $5 ; t=-0.760, P=0.453$ ), indicating that the reduction in hyperinflation might be caused by PDE4 inhibition. Subsequently, it can be observed that lobes that reduce during hyperinflation after treatment are better ventilated (Figure 6; $t=-5.368, P<0.001$ ), where ventilation is determined by the difference in TLC and FRC lobe volumes. FRI-based aerosol deposition shows that enhanced ventilation also leads to more particle deposition of ICSs/LABAs/ LAMAs in the better-ventilated areas (Figure 7; $t=2.407$, 


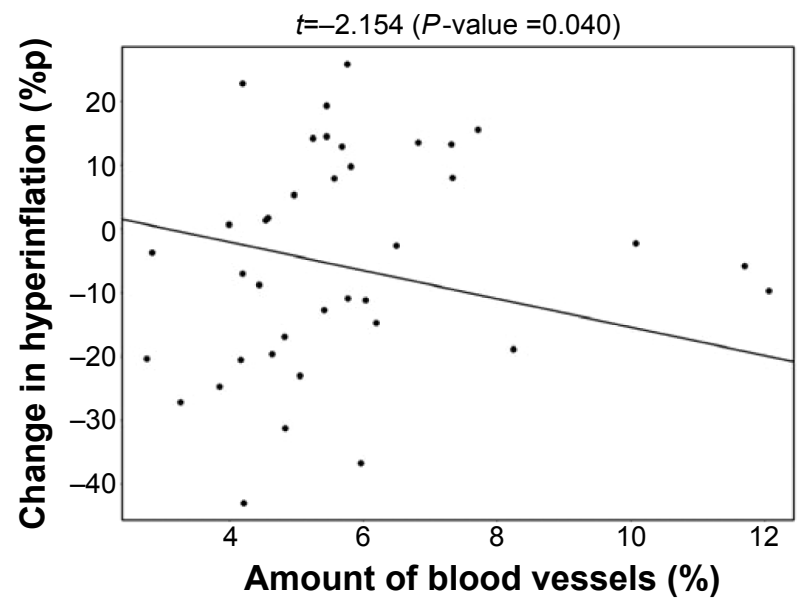

Figure 4 Negative correlation between the lobar blood vessel density and the change in lobar hyperinflation in the roflumilast responder group after 6 months of treatment.

Note: Better-perfused lobes experience larger reduction in hyperinflation.

Abbreviation: \%p, percentage of predicted.

$P=0.024)$. Finally, the study shows that areas receiving more particles have increased FRI-based bronchodilation (Figure 8; $t=2.564, P=0.017$ ), leading to an increase in $\mathrm{FEV}_{1}$ (Figure 9, $R=0.348, P=0.029$ ).

\section{Discussion}

This report provides additional analyses of the study reported by De Backer et al, ${ }^{10}$ with the main focus on regional changes in ventilation, aerosol deposition, bronchodilation, and blood vessel density. Lobes with a high percentage of blood vessels reduce more during hyperinflation in the responder group.

For the first time, HRCT data and CFD are used to understand the interaction between systemic and inhaled drugs. The study appears to support the hypothesis that roflumilast reaches, via the vasculature, areas that were

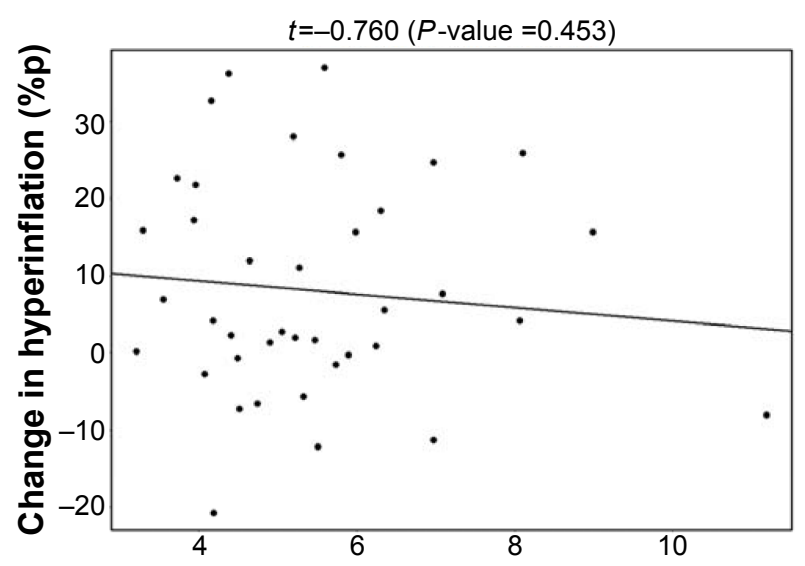

Amount of blood vessels (\%)

Figure $\mathbf{5}$ No correlation between the lobar blood vessel density and the change in lobar hyperinflation in the placebo group.

Abbreviation: \%p, percentage of predicted.

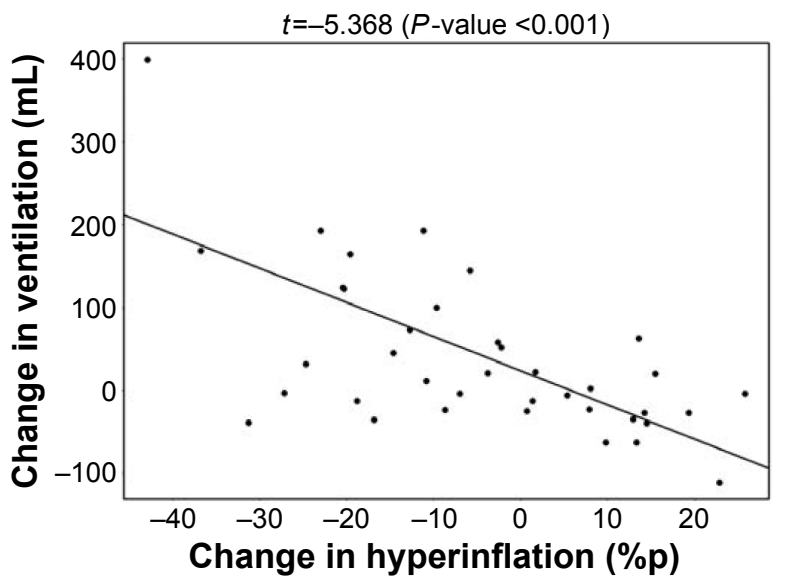

Figure 6 Negative correlation between the change in lobar hyperinflation and the change in lobar ventilation in the Roflumilast responder group after 6 months of treatment.

Note: Lobes that experience a larger reduction in hyperinflation are better ventilated.

Abbreviation: \%p, percentage of predicted.

previously undertreated by the inhaled medication. Opening smaller airways or preventing airway collapse, presumably by reducing inflammation and edema, results in a reduction of regional hyperinflation, eventually cascading into enhanced efficacy of the concomitant ICS/LAMA/LABA treatment. Even though this appears to be the mode of action of roflumilast, it is likely that the PDE4 inhibitor by itself also has a positive, but potentially smaller effect, on the larger airways in terms of bronchodilation and reduction of inflammation. This can be concluded from the differences observed between the treatment and placebo arms. The latter remains a topic of ongoing research and can be assessed by studying inhaled PDE4 inhibitors currently under development ${ }^{18,19}$ and

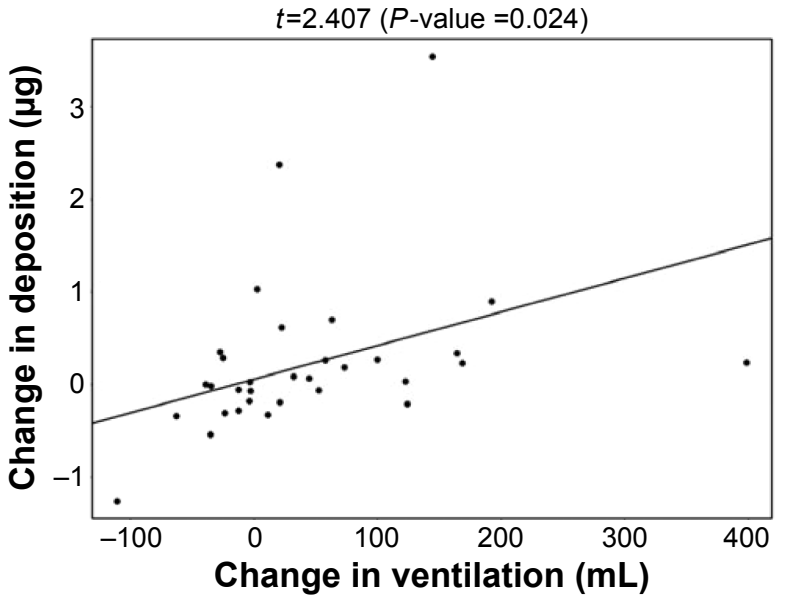

Figure 7 Positive correlation between the change in lobar ventilation and the change in lobar aerosol deposition in the roflumilast responder group after 6 months of treatment.

Note: Lobes that are better ventilated consequently experience more drug deposition. 


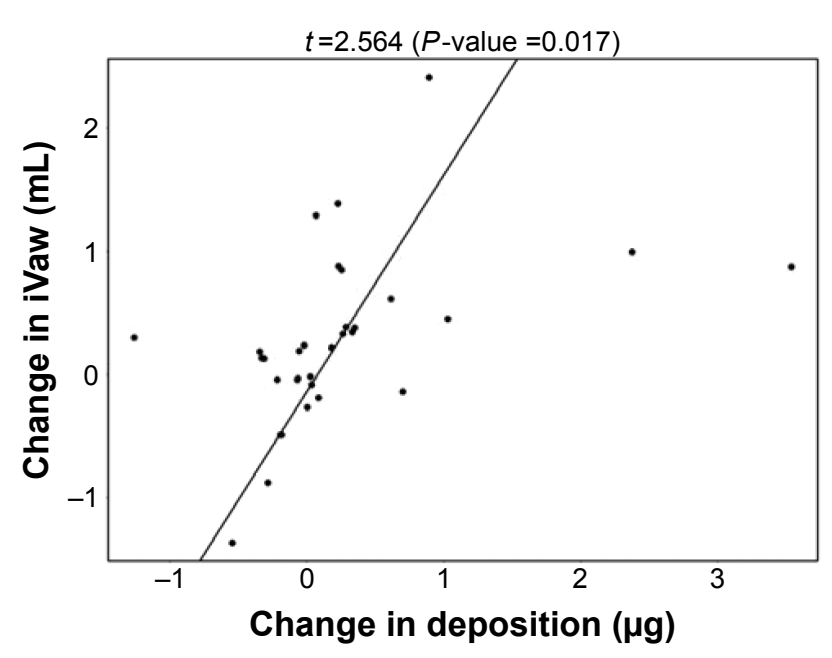

Figure 8 Positive correlation between the change in lobar aerosol deposition and the change in lobar CT-based bronchodilation (iVaw) in the roflumilast responder group after 6 months of treatment.

Note: Lobes with higher deposition had more bronchodilation.

Abbreviations: CT, computed tomography; iVaw, image-based airway volume.

comparing the results with those from orally administered roflumilast. Overall, the results of this study raise several challenging research questions.

A previous study by Vestbo et $\mathrm{al}^{32}$ on inhaled PDE4 inhibitor showed no effect of treatment on spirometric measures and symptoms score in patients with moderateto-severe COPD. However, we think this needs further investigation: It may have no effect on spirometric values, but we can get better insight of the local responses with functional respiratory imaging to fully evaluate the regional hyperinflation, internal airflow distribution, regional aerosol deposition patterns, and image-based bronchodilation. So, inhaled roflumilast could be a therapeutic option for COPD

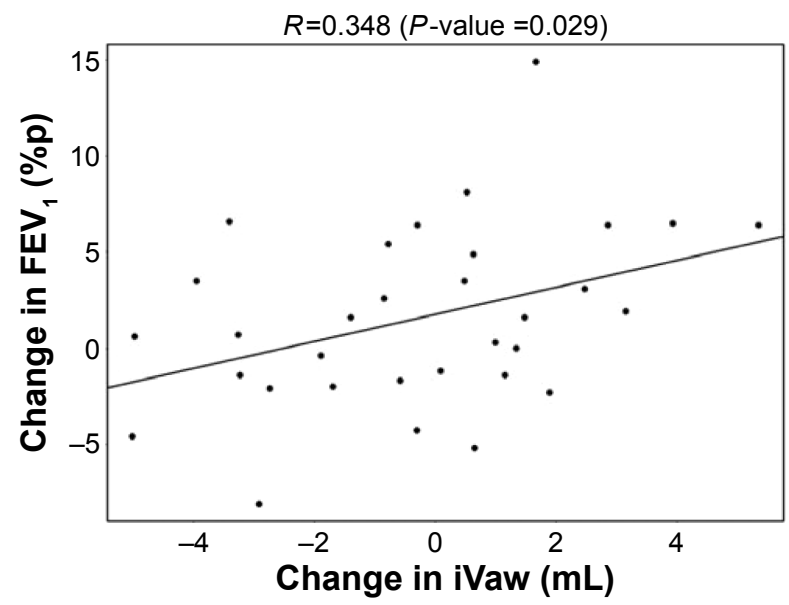

Figure 9 Positive correlation between the change in CT-based bronchodilation (iVaw) and the observed changes in $\mathrm{FEV}_{1}$.

Note: More bronchodilation leads to better FEV,

Abbreviations: CT, computed tomography; $\mathrm{FEV}_{1}$, forced expiratory volume in I second; iVaw, image-based airway volume; \%p, percentage of predicted. patients without the systemic side effects, but it needs further investigation.

First of all, one can wonder whether inhalation medication by itself adequately treats COPD patients. Today's standard of care in lung diseases is usually LABA, LAMA, ICS, or a combination thereof. However, if it is really true that areas in the lungs are chronically undertreated due to either ineffective deposition (insufficient amount of particles) or ineffective exposure (insufficient dose), then even the most potent compounds will not be able to demonstrate high efficacy. Giembycz and Newton ${ }^{20}$ recently argued that roflumilast is beneficial in severe COPD patients who frequently show exacerbation because these patients are prone to bacterial colonization, infection, and a high level of inflammation. Moreover, Yu et $\mathrm{al}^{21}$ concluded that patients at high risk of severe exacerbations have a net benefit from roflumilast. On the basis of these findings and the results of our study, we would suggest that frequent exacerbations in patients are a result of inadequate control of the disease using inhalation medication due to low regional exposure of the drug in poorly ventilated areas. The systemic delivery route of roflumilast, and particularly earlier start of the treatment, therefore could mitigate this undertreatment and provide a benefit to these severe patients. While the results of this study provide initial evidence of the hypothesis, additional confirmatory trials are needed. Considering this, the recent publication of the REACT trial results ${ }^{22}$ strengthens the belief of the authors that the proposed hypothesis might indeed be correct.

The second interesting research topic relates to the pathophysiology of COPD exacerbations. The label of roflumilast currently indicates that the product can be used to reduce exacerbations, and the studies mentioned herein also support this indication. Our trial demonstrated that the mode of action of roflumilast is probably related to regional hyperinflation. This finding triggers the following hypothesis: COPD exacerbations are associated with regional hyperinflation. The association of exacerbations with overall hyperinflation measured using body plethysmography has been described in recent studies. ${ }^{23,24}$ However, these aggregate measures might not fully reflect the changes in hyperinflation due to the interdependence between the lobes. It could be that certain lobes are hyperinflated, but when an intervention reduces the hyperinflation in that lobe, the neighboring lobe(s) might reinflate, thereby reducing the overall signal in the conventional black box parameters. Imaging, and in particular FRI, provides highly detailed, quantifiable information, which makes it a valuable tool in early clinical development of novel compounds. The fact 
that FRI end points can be assessed on a lobar level already provides higher statistical power because one patient now yields five measurement points, compared to conventional outcome parameters such as $\mathrm{FEV}_{1}$, whereby one patient only yields one black box measure. However, a correction for the lobe interdependence needs to be included in the statistical analysis (as described in the preceding text, via the generalized estimating equation with an autoregressive covariance). In a recent review, Coxson et $\mathrm{al}^{25}$ confirmed the potential of imaging to go beyond $\mathrm{FEV}_{1}$ in COPD.

Especially in early clinical phases, it is particularly useful to have a surrogate for exacerbations. Having a surrogate would allow the researcher to assess the effect of the novel drug on this surrogate, thereby de-risking the late phase of drug development.

At present, the usefulness of FRI to better characterize COPD exacerbations is speculative, and additional studies using FRI need to be performed to confirm the correlation between regional hyperinflation and exacerbations. A clinical trial with the latter topic (NCT01684384) has been recently concluded, and the results will be available shortly.

Often, in clinical trials with FRI and spirometry, one raises the following question: which end points are "measurements" and which end points are "calculations". While the easy answer is to state that all parameters derived from the HRCT are calculated, the topic becomes more complex when we take one step back and realize that virtually all measurements are in fact calculations. The $\mathrm{FEV}_{1}$, for instance, is a calculation of a pressure drop over a known resistance integrated between 0 second and 1 second; the HRCT is regarded as a measurement or even a picture, while it is inherently a calculation (the $\mathrm{C}$ stands for computed). The problem we experienced using terms such as calculation, model, simulation etc is that these end points are often dismissed as not real or idealized, while the opposite is true. FRI parameters are advanced quantifications of patientspecific measures. In our view, the difference between a "measurement" and a "calculation" is merely the level of general acceptance of the method used to obtain the parameter.

The study demonstrated that orally administered roflumilast supports the reduction of regional hyperinflation in areas previously undertreated by inhalation medication. The local reduction in hyperinflation induces a redistribution of ventilation and aerosol deposition, leading to enhanced efficacy of the concomitant ICS/LABA/LAMA therapy. FRI appears to be a sensitive tool to describe the mode of action of novel compounds in COPD. Future studies need to confirm the enhanced sensitivity and the potential of FRI parameters to act as surrogates for clinically relevant, but more difficult to measure, end points such as exacerbations.

\section{Acknowledgment}

The study was funded by Takeda Pharmaceutical Company.

\section{Disclosure}

The authors have no other relevant affiliations or financial involvement with any organization or entity with a financial conflict with the subject matter or materials discussed in the manuscript apart from those disclosed.

\section{References}

1. Solèr M. Omalizumab for severe allergic asthma: 7 years and open questions. Respiration. 2014;88(2):158-161.

2. Wenzel S, Ford L, Pearlman D, et al. Dupilumab in persistent asthma with elevated eosinophil levels. N Engl J Med. 2013;368(26):2455-2466.

3. De Backer JW, Vos WG, Vinchurkar SC, et al. Validation of computational fluid dynamics in CT-based airway models with SPECT/CT. Radiology. 2010;257(3):854-862.

4. Vinchurkar S, Backer LD, Vos W, Holsbeke CV, Backer JD, Backer WD. A case series on lung deposition analysis of inhaled medication using functional imaging based computational fluid dynamics in asthmatic patients: effect of upper airway morphology and comparison with in vivo data. Inhal Toxicol. 2012;24(2):81-88.

5. de Rochefort L, Vial L, Fodil R, et al. In vitro validation of computational fluid dynamic simulation in human proximal airways with hyperpolarized $3 \mathrm{He}$ magnetic resonance phase-contrast velocimetry. J Appl Physiol (1985). 2007;102(5):2012-2023.

6. De Backer JW, Vos WG, Devolder A, et al. Computational fluid dynamics can detect changes in airway resistance in asthmatics after acute bronchodilation. J Biomech. 2008;41(1):106-113.

7. De Backer LA, Vos W, De Backer J, Van Holsbeke C, Vinchurkar S, De Backer W. The acute effect of budesonide/formoterol in COPD: a multi-slice computed tomography and lung function study. Eur Respir J. 2012;40(2):298-305.

8. De Backer LA, Vos WG, Salgado R, et al. Functional imaging using computer methods to compare the effect of salbutamol and ipratropium bromide in patient-specific airway models of COPD. Int J Chron Obstruct Pulmon Dis. 2011;6:637-646.

9. De Backer J, Vos W, Van Holsbeke C, et al. Effect of high-dose $\mathrm{N}$-acetylcysteine on airway geometry, inflammation, and oxidative stress in COPD patients. Int J Chron Obstruct Pulmon Dis. 2013;8: 569-579.

10. De Backer W, Vos W, Van Holsbeke C, et al. The effect of roflumilast in addition to LABA/LAMA/ICS treatment in COPD patients. Eur Respir J. 2014;44(2):527-529.

11. Chong J, Poole P, Leung B, Black PN. Phosphodiesterase 4 inhibitors for chronic obstructive pulmonary disease. Cochrane Database Syst Rev. 2011;(5):CD002309.

12. Bateman ED, Rabe KF, Calverley PMA, et al. Roflumilast with long-acting $\beta 2$-agonists for COPD: influence of exacerbation history. Eur Respir J. 2011;38(3):553-560.

13. Rabe KF. Roflumilast for the treatment of chronic obstructive pulmonary disease. Expert Rev Respir Med. 2010;4(5):543-555.

14. Fabbri LM, Calverley PMA, Izquierdo-Alonso JL, et al; M2-127 and M2-128 Study Groups. Roflumilast in moderate-to-severe chronic obstructive pulmonary disease treated with longacting bronchodilators: two randomised clinical trials. Lancet. 2009;374(9691):695-703.

15. Calverley PMA, Rabe KF, Goehring U-M, et al; M2-124 and M2-125 Study Groups. Roflumilast in symptomatic chronic obstructive pulmonary disease: two randomised clinical trials. Lancet. 2009; 374(9691):685-694.

16. De Backer JW, Vos WG, Vinchurkar SC, et al. Validation of computational fluid dynamics in CT-based airway models with SPECT/CT. Radiology. 2010;257(3):854-862. 
17. Janssens W, Liu Y, Liu D, et al. Quality and reproducibility of spirometry in COPD patients in a randomized trial (UPLIFT $\left({ }^{\circledR}\right)$ ). Respir Med. 2013;107(9):1409-1416.

18. Franciosi LG, Diamant Z, Banner KH, et al. Efficacy and safety of RPL554, a dual PDE3 and PDE4 inhibitor, in healthy volunteers and in patients with asthma or chronic obstructive pulmonary disease: findings from four clinical trials. Lancet Respir Med. 2013;1(9):714-727.

19. Watz H, Mistry SJ, Lazaar AL. Safety and tolerability of the inhaled phosphodiesterase 4 inhibitor GSK256066 in moderate COPD. Pulm Pharmacol Ther. 2013;26(5):588-595.

20. Giembycz MA, Newton R. How phosphodiesterase 4 inhibitors work in patients with chronic obstructive pulmonary disease of the severe, bronchitic, frequent exacerbator phenotype. Clin Chest Med. 2014;35(1): 203-217.

21. Yu T, Fain K, Boyd CM, et al. Benefits and harms of roflumilast in moderate to severe COPD. Thorax. 2014;69(7):616-622.

22. Martinez FJ, Calverley PMA, Goehring U-M, Brose M, Fabbri LM, Rabe KF. Effect of roflumilast on exacerbations in patients with severe chronic obstructive pulmonary disease uncontrolled by combination therapy (REACT): a multicentre randomised controlled trial. Lancet. 2015;385(9971):857-866.

23. Wedzicha JA, Brill SE, Allinson JP, Donaldson GC. Mechanisms and impact of the frequent exacerbator phenotype in chronic obstructive pulmonary disease. BMC Med. 2013;11:181.

24. Wedzicha JA, Decramer M, Seemungal TAR. The role of bronchodilator treatment in the prevention of exacerbations of COPD. Eur Respir J. 2012;40(6):1545-1554.
25. Coxson HO, Leipsic J, Parraga G, Sin DD. Using pulmonary imaging to move COPD beyond FEV1. Am J Respir Crit Care Med. 2014; 190(2):135-144.

26. Chapman KR, Fogarty CM, Peckitt C, et al. Delivery characteristics and patients' handling of two single-dose dry-powder inhalers used in COPD. Int J Chron Obstruct Pulmon Dis. 2011;6:353-363.

27. Weuthen T, Roeder S, Brand P, Müllinger B, Scheuch G. In vitro testing of two formoterol dry powder inhalers at different flow rates. $J$ Aerosol Med. 2002;15(3):297-303.

28. Hoe S, Traini D, Chan H-K, Young PM. Measuring charge and mass distributions in dry powder inhalers using the electrical next generation impactor (eNGI). Eur J Pharm Sci. 2009;38(2):88-94.

29. Tarsin WY, Pearson SB, Assi KH, Chrystyn H. Emitted dose estimates from Seretide ${ }^{\circledR}$ Diskus ${ }^{\circledR}$ and Symbicort ${ }^{\circledR}$ Turbuhaler ${ }^{\circledR}$ following inhalation by severe asthmatics. Int J Pharm. 2006;316(1-2):131-137.

30. De Backer W, Devolder A, Poli G, et al. Lung Deposition of BDP/ Formoterol HFA pMDI in healthy volunteers, asthmatic, and COPD patients. J Aerosol Med Pulm Drug Deliv. 2010;23(3):137-148.

31. Trukhacheva LA, Gorpinchenko NV, Dementyev SP. Comparative analysis of the in vitro equivalence of the metered aerosol inhalers Seretide and Tevacomb conducted by the new generation impactor next. Pediatr Pharmacol. 2012;9(5):70-74.

32. Vestbo J, Tan L, Atkinson G, Ward J; UK-500,001 Global Study Team. A controlled trial of 6-weeks' treatment with a novel inhaled phosphodiesterase type-4 inhibitor in COPD. Eur Respir J. 2009;33(5): 1039-1044.
International Journal of COPD

\section{Publish your work in this journal}

The International Journal of COPD is an international, peer-reviewed journal of therapeutics and pharmacology focusing on concise rapid reporting of clinical studies and reviews in COPD. Special focus is given to the pathophysiological processes underlying the disease, intervention programs, patient focused education, and self management protocols.

\section{Dovepress}

This journal is indexed on PubMed Central, MedLine and CAS. The manuscript management system is completely online and includes a very quick and fair peer-review system, which is all easy to use. Visit http://www.dovepress.com/testimonials.php to read real quotes from published authors. 